\title{
REVIEW
}

\section{Stratified medicine: drugs meet genetics}

\author{
Ian P. Hall
}

\begin{abstract}
It is well recognised that genetic factors play a major role in the development of respiratory diseases such as asthma and chronic obstructive pulmonary disease. However, whilst extensive data exist on diseases caused primarily by single gene defects, such as $\alpha_{1}$-antitrypsin deficiency, the genetic factors responsible for the development of complex disease are only now being defined. Once the gene(s) responsible for the heritable element of disease risk are known, the next step is to identify the mechanisms underlying the pathophysiological effects of the causal mutations in these genes. This process can be time consuming, but allows a full understanding of the mechanisms underlying disease development to be obtained. This knowledge can then potentially be used to stratify patient groups within (or even across) disease boundaries and then to target therapy more effectively.
\end{abstract}

KEYWORDS: Asthma, chronic obstructive pulmonary disease, cystic fibrosis, genetics, lung cancer, lung function

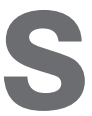
tratified medicine involves the use of patientspecific features to determine groups of patients who might respond well or badly to a given medication. The factors which can be used to aid this process are varied; typically this might include the use of specific clinical features, biomarkers or, increasingly, genetic information. There has been a huge expansion in the amount of genetic data in the public domain since the completion of the human genome project just over 10 yrs ago. However, there is a long time-lag between the identification of genetic factors underlying specific diseases and the use of this information to alter treatment strategies for patients. A cynical viewpoint would be that the genetic information becoming available has therefore been of minimal value given the huge investment made in this field, but a more rational viewpoint would be that this knowledge will increasingly be used as an aid in patient management.

There are a number of ways in which genetic information could be used in clinical medicine to modify therapeutic approaches. First, genetic information can be used directly to alter treatment for a patient, either to inform adjustment of drug dosage to avoid side-effects, or to select a given treatment from a range of possible alternatives based on the genetic profile of the patient. This approach is sometimes called pharmacogenetics, or personalised medicine. There is an assumption that this approach is new but actually the concept of using a stratified approach to individualise treatment is at least 2,000 yrs old. In ancient Greece, the Favists, a sect which numbered Pythagoras amongst its members, followed a number of rules including the avoidance of eating beans (fava being the Latin name for the broad bean genus). Ingestion of beans subsequently was found to precipitate exacerbations of disease in patients with glucose-6phosphate dehydrogenase (G6PD) deficiency, a condition characterised by episodes of haemolytic anaemia upon exposure to precipitants. The genetic mechanisms underlying this condition are now well understood. The gene for G6PD is on the $X$ chromosome and the major mutations responsible for causing disease have been defined (e.g. Arg459Leu and Arg463His). Individuals from many Mediterranean countries (where G6PD deficiency is more common than in northern European countries) are screened at birth for the condition using a nicotinamide adenine dinucleotide phosphate fluorescence assay, and are advised of the risk following exposure to known precipitants of crises. Precipitants include drugs such as nitrofurantoin, aspirin and isoniazid.

\section{STRATIFIED MEDICINE AND TARGETED THERAPY}

A second potential use of genetic information is to define disease sub-phenotype, which will respond differently to a given treatment. In concept, the use of genetic information in this context is no different from using other measurements such as total immunoglobulin $\mathrm{E}$ to guide omalizumab therapy in patients with difficult-to-control asthma. The best examples of this approach come from the oncology field.
AFFLLIATION

Division of Therapeutics and Molecular Medicine, Queen's Medical Centre, Nottingham, UK.

\section{CORRESPONDENCE}

I.P. Hall

Division of Therapeutics and

Molecular Medicine

D floor south block

Queen's Medical Centre

Nottingham

NG7 2UH

UK

E-mail: lan.Hall@nottingham.ac.uk

Received

Dec 202012

Accepted after revision:

Jan 032012

\section{PROVENANCE}

Publication of this peer-reviewed

article was supported by Vertex Pharmaceuticals Inc., USA (principal sponsor, European Respiratory Review issue 127). 
In the respiratory disease area, genetic information about tumour profiling is increasingly being used in the management of nonsmall cell lung cancer (NSCLC). Whilst overall the response of NSCLC to chemotherapy remains generally disappointing, there are some markers that can be used to optimise therapy. Two examples are therapy targeting the epidermal growth factor receptor (EGFR) and therapy targeting the ELM4-ALK fusion protein. Gefitinib and erlotinib are both agents which are effective only in patients whose tumours harbour specific EGFR profiles, and hence their use is limited to treatment of patients proven to have these specific tumour profiles $[1,2]$. There remain questions about the best way to target therapy defined by tumour genotype. In particular, there are concerns that tumour genotypes are not uniform across all cells within a tumour and that, therefore, if tumour cell clones exist with different mutation profiles these will expand and the tumour will become resistant to treatment. Nonetheless, the use of tumour profiling has become the subject of guidelines [3]. In addition, the number of patients with mutations that allow this approach may be relatively small; for example, the use of crizotinib, which specifically targets the ELM4-ALK mutation in NSCLC, is limited to $\sim 5 \%$ of tumours $[4,5]$. This also raises issues in terms of the cost-effectiveness of screening for relatively rare responder groups.

The previous examples relate to tumour genotype rather than host genotype but, increasingly, subgroups of patients within disease groups whose disease is driven by a specific genetically determined mechanism are being identified. One excellent example of this from the non-respiratory field is in neonatal diabetes mellitus. A number of infants presenting in the early post-natal period with diabetes carry activating mutations in KCNJ11, the gene which codes for the adenosine triphosphatesensitive $\mathrm{K}^{+}$channel Kir6.2 [6]. Activation of this channel inhibits insulin secretion and, therefore, patients with activating mutations present early in life with hyperglycaemia. Until recently, these patients required treatment with insulin therapy. However, gaining an understanding of the genetic mechanism underlying the development of diabetes in this group of patients led to the realisation that activation of this channel could be bypassed in these individuals by administering sulfonylureas, such as glibenclamide. This is because sulfonylureas act at a different site to inhibit channel activation. Transferring patients with early onset diabetes caused by this mechanism onto sulfonylureas led to sustained improvement in markers of disease control, such as glycosylated haemoglobin $(\mathrm{HbA} 1 \mathrm{c})$, and has obvious benefits in terms of quality of life for these infants and their families [7].

Until recently, examples in which patients with respiratory disease could be stratified in this way were absent. One of the first respiratory diseases where an understanding of the genetic basis for disease development became available is $\alpha_{1}$-antitrypsin deficiency. Although $\alpha_{1}$-antitrypsin deficiency has been recognised for several decades as a disease entity, replacement therapy for this group of patients affected by early onset emphysema has been rather disappointing in terms of altered clinical outcomes.

However, a paradigm shifting example is now available to the scientific community interested in respiratory medicine. This relates to stratified approaches to the treatment of cystic fibrosis (CF).

\section{CF AND STRATIFIED MEDICINE}

CF occurs in patients carrying two deleterious mutations in the $\mathrm{CF}$ transmembrane regulator protein (CFTR). The CFTR gene was identified in 1989, and current databases include around 1,000 different mutations. These fall into several different classes; for more information refer to the article by DERICHS [8] in this issue. The identification of the gene for CFTR led to hopes for improved therapy for this condition, and gene therapy trials started early in the 1990s. However, poor efficacy of the transgene when delivered by nonviral vectors or adverse events when gene therapy was performed using adenoviral vectors has prevented this approach being used outside of clinical trial settings. Thus, alternative approaches have been developed to try and bypass the mechanisms underlying defective CFTR function. A small subgroup of patients was identified carrying gating channel mutations, of which the most common is the G551D mutation. Because individuals with only one copy of the CFTR gene carrying a mutation are essentially normal, treatment can be directed at individuals heterozygous for CFTR mutations who carry a gating channel mutation in combination with other mutations, of which delta F508 is most common in the European population. A classical high throughput small molecule screen led to the identification of lead compounds able to reverse the CFTR gating channel mutation effect, and this in turn led to the clinical development of the drug ivacaftor. In a recently reported clinical trial, treatment with this agent produced sustained, clinically relevant improvements in forced expiratory volume in $1 \mathrm{~s}$ (FEV1) and also improved markers of CFTR function such as sweat sodium [9]. These data are discussed in detail elsewhere. It is worth considering, however, the implications of this approach. It is probable that ivacaftor would also benefit patients with some of the other rarer gating channel mutations (e.g. G178R, G551S, G970R, G1244E, S1255P and G1349D); however, undertaking a clinical trial in this group would be impractical due to the rarity of the mutations. In addition, whilst the G551D mutation is part of the panel of variants screened for in many centres, these other gating channel mutations are not on the panels used in most centres. This raises the issue of the need to develop suitable screening assays for rare CFTR mutations. Whilst this could be undertaken using the same kind of panels currently utilised, it may prove more cost-effective in the future to use next generation sequencing approaches to sequence the whole of the CFTR gene region in all affected individuals. However, whilst the technology is available in many research laboratories, the use of deep sequencing in clinical diagnostic laboratories is not widely established and has significant resource, quality control and training implications.

\section{STRATIFIED MEDICINE AND CHRONIC OBSTRUCTIVE PULMONARY DISEASE}

The major question in the field of stratified medicine that needs addressing is how generalisable this kind of approach will prove to be. Whilst the case for stratification of CF patients for consideration for ivacaftor seems clear, whether or not similar approaches will prove effective in common respiratory diseases, such as asthma and chronic obstructive pulmonary disease (COPD), remains to be determined. Again, there is some cynicism about the use of genetic information to define sub-phenotypes with specific mechanisms that could be 
targeted. However, the state of knowledge regarding the genetics of these common conditions is much less well developed, and it is important to remember the time lag required from the discovery of the gene to bringing successful strategies to the clinic in $\mathrm{CF}$.

To address this issue further, it is worth considering the advances which have been made recently in trying to unravel the genetic basis of COPD. As mentioned previously, one subgroup of patients with early onset emphysema was identified many years ago based upon their $\alpha_{1}$-antitrypsin status; however, until recently little was known about the genetic basis of COPD in the majority of patients. A number of large scale studies using genome-wide association approaches have thrown light on this issue. The largest of these have used pre-bronchodilator FEV1 and FEV1/forced vital capacity (FVC) as their main end-points; however, as airflow obstruction is a key feature of COPD it is perhaps not surprising that a number of the genes found to contribute to variability in spirometry have also proven to contribute to COPD risk per se. Table 1 includes a list of genes identified by a series of meta-analyses by the Spirometa and CHARGE (Cohort for Heart and Aging Research in Genomic Epidemiology) consortia which contribute to either FEV1 or FEV1/FVC ratio; those in bold have also been shown to contribute to COPD risk [10-14]. Because the genome-wide association studies of COPD have been substantially smaller than those looking at spirometry at a population level, the ability to identify signals is likely to be smaller, and it follows that an increasing number of the genes found to contribute to spirometric indices will also be found to contribute to COPD risk over the next few years.

The really interesting observations about the data which have been generated using these approaches is that the genes that have been identified in general are not those whose products have historically been thought to be important in the pathophysiology of COPD. In the past, there have been many candidate gene studies looking at possible genetic contributions

\begin{tabular}{ll} 
TABLE 1 Genes/loci associated with spirometric indices of \\
lung function \\
MF & CFDP1 \\
AP2 & KCNE2 \\
Near TGFB2 & TNS1 \\
HDAC4 & THSD4 \\
RARB & GSTCD \\
MECO & HTR4 \\
SPATA9 & AGER \\
ARMC2 & HHIP \\
NCR3 & FAM13A \\
ZKSCAN3 & ADAM19 \\
CDC123 & GPR126 \\
C10orf11 & PITCH1 \\
LRP1 & IREB2/CHRNA3/CHRNA5 \\
CCDC38 & \\
MMP15 & \\
\hline
\end{tabular}

Those genes/loci shown in bold have also been shown to be associated with chronic obstructive lung disease per se. For more details refer to [10-14]. to lung function or COPD risk, and the lack of replication of the findings from these generally small studies in the large genomewide association studies suggests many of the candidate gene findings are false positives. This observation is backed up by a study we performed based on a "look up" of all previous candidate gene studies identified in a systematic literature review, in which no p-values were seen greater than one would expect by chance given the number of genes previously examined in these studies [15]. It is still possible that some of these previously identified genes may play a role, in that there may be rarer variants in these genes which are not well captured by the single nucleotide polymorphisms used on the genomewide association platforms. Nonetheless, it appears that the robust signals identified in meta-analyses of large population studies imply a key role in lung function determination of a range of novel genes, many of which appear to play a role in lung development.

The second observation of interest is that the genetic effects seen to date appear to be largely independent of smoking exposure. Even the interaction between smoking status and lung function, which is seen at the CHRN3/5 locus, is partly explained by smoking behaviour alone, in that this locus appears to determine, in part, the number of cigarettes smoked per day, although interestingly a small effect of this locus on the risk of developing airflow obstruction was also seen in nonsmokers [14]. The methods used to look for interactions in genome-wide association study datasets are still being developed, and it may be that some signals exist that current methods are inadequate to identify; however, it seems unlikely that there are large gene-by-smoking interactions, which explain a substantial proportion of genetic risk of lung disease in smokers. Rather, the data suggest that genetic contributors to lung function determination work separately (and additively) with smoking exposure to dictate risk of developing airflow obstruction. It is increasingly recognised that, particularly in elderly populations, there are individuals who have not smoked who fulfil criteria for having a diagnosis of COPD, and it may be that this occurs in individuals who carry more genetic risk factors than the rest of the population.

There are likely to be many more genes identified that contribute to lung function and COPD, as the variability explained by the currently known genetic signals is $<10 \%$ of the total variability in FEV1 in the general population. The key question remains, however, and this is what benefit can be obtained from the identification of these genetic-association signals? The initial requirement is to define the functional contribution of these genes and the way in which lung function is altered by the key polymorphisms. This can be quite challenging, and might, for example, require the development of specific transgenic animal models or the use of recombinant cell lines as well as the study of tissue from individuals of known genotype.

As well as the possibility of the products of these genes being useful therapeutic targets, one interesting prospect is whether or not these genes can be used in a clinically relevant setting for risk prediction. This approach could potentially be of benefit in two ways. First, overall risk of COPD could be predicted using a model including age, population risk, smoking status and genetics; this would allow a risk score to 
TABLE 2 Examples of drug label recommendations

\begin{tabular}{|c|c|c|c|}
\hline Gene & Example drugs & Effect of polymorphism & Regulatory recommendation \\
\hline \multirow[t]{2}{*}{ CYP2C19 } & Clopidogrel & $\begin{array}{l}\text { Reduced metabolism of clopidogrel, lower } \\
\text { exposure to active metabolite and, thus, } \\
\text { higher cardiovascular risk }\end{array}$ & $\begin{array}{l}\text { Warning of potential effect; consider alternative } \\
\text { treatment }\end{array}$ \\
\hline & Voriconazole & $\begin{array}{l}\text { Reduced metabolism of voriconazole, thus } \\
\text { increased drug exposure }\end{array}$ & Warning of potential effect \\
\hline \multirow[t]{2}{*}{ CYP2D6 } & Codeine & Leads to ultra-rapid metabolism to active metabolite & Warning of potential effect; use lowest effective dose \\
\hline & Tamoxifen & $\begin{array}{l}\text { Poor metabolisers have higher plasma } \\
\text { concentrations }\end{array}$ & Warning of potential effect \\
\hline \multirow[t]{2}{*}{ EGFR } & Gefitinib & $\begin{array}{l}\text { Effectiveness depends on EGFR status of tumour in } \\
\text { nonsmall cell lung cancer }\end{array}$ & Tumour profiling to guide therapy \\
\hline & Erlotinib & & \\
\hline HER 2 & Trastuzimab & $\begin{array}{l}\text { Effect depends on HER2 expression levels in } \\
\text { breast cancer }\end{array}$ & Screen for HER2 expression before treatment \\
\hline \multirow[t]{2}{*}{ HLA-B*1502 } & Carbamazepine & $\begin{array}{l}\text { Greatly increased risk of severe dermatological } \\
\text { hypersensitivity reaction }\end{array}$ & $\begin{array}{l}\text { Screen patients in high-risk ethnic groups to } \\
\text { exclude genotype before commencement }\end{array}$ \\
\hline & Abacavir & $\begin{array}{l}\text { Greatly increased risk of generalised hypersensitivity } \\
\text { reaction }\end{array}$ & Screen all patients prior to drug commencement \\
\hline LDLR & Atorvastatin & $\begin{array}{l}\text { LDL receptor deficiency or absence leads to familial } \\
\text { hypercholesterolaemia }\end{array}$ & Dose adjustments in genetic risk groups \\
\hline NAT & Rifampicin & Slow metabolism and greater drug exposure & Warning of potential effect \\
\hline TPMT & Azathioprine & $\begin{array}{l}\text { Slower metabolism and greater resultant risk of } \\
\text { myelotoxicity }\end{array}$ & Screen all patients prior to drug commencement \\
\hline
\end{tabular}

EGFR: epidermal growth factor receptor; LDL: low-density lipoprotein; UCD: urea cycle disorders; SNP: single nucleotide polymorphism. Adapted from [16].

be developed similar to the scores used in cardiovascular prevention in the primary care sector. The main intervention which could benefit from this approach would be to target smoking cessation approaches. Obviously, the risk of this approach is that if an individual fell into a low-risk (of developing COPD) group they might feel empowered to continue smoking, but careful use of anti-smoking advice could mitigate this. The approach is not dissimilar to the use of cholesterol measurements in cardiovascular risk prediction/ intervention, there is probably no cholesterol level where individuals would not benefit from intervention to produce a further lowering of cholesterol levels, but intervention is targeted at those at highest risk.

The second possibility is that genetic factors could be used to identify sub-phenotypes of COPD which might respond differently. At present this approach remains hypothetical but is clearly of potential interest. Whilst many of the genes identified to date appear to lie in developmental pathways, and thus may prove difficult to use in this way, some of the genes being identified do tag pathways which may be driving inflammatory responses in subgroups of patients. Similar approaches to those described above are being pursued in most other common respiratory diseases, with the asthma field being probably the most advanced.

\section{IMPLICATIONS OF STRATIFIED MEDICINE}

Finally, it is important to realise the challenges inherent is the advent of stratified medicine developments. These are many and varied. Most doctors currently in practice went to medical school at a time before the use of the genetic methods discussed in this review had been developed, and so there is a major educational and training challenge in the adoption of stratified medicine. Laboratories will need to adopt new techniques and have rigorous quality-control mechanisms in place to ensure accurate genetic information is available to use in clinical decision making. The case for cost-effectiveness of stratified approaches will need to be convincing for these approaches to be introduced into standard clinical practice. 
At present, the use of stratified medicine to target therapy is likely to be primarily located in academic centres with a specialist interest in the area, but as costs fall and the number of examples where patient outcomes are improved increases there will be wider adoption. The speed of technical advance, particularly in sequencing technologies, has been extraordinary, and with the cost of whole genome sequencing falling to a few thousand dollars there will be a huge increase in the available datasets to help develop these approaches. This will bring inevitable ethical challenges but, in principle, the use of genetic data is little different from the use of many other standard biochemical data currently generated as part of routine clinical care. Regulatory guidance will be needed from agencies such as the US Food and Drug Administration. A number of drugs already have information on regulatory websites about the potential to use genetic testing to guide prescribing (table 2) [16]. Therefore, I predict that there will be gradual but increasing adoption of stratified approaches using genetic information in the management of patients with both rare and common diseases over the next 10-15 yrs.

\section{STATEMENT OF INTEREST}

I.P. Hall received a speaker's fee for participating in the sponsored symposium at the 2012 European Respiratory Society Congress. Work in I.P. Hall's laboratory relevant to the content of this review has been funded by the Medical Research Council, Asthma UK and Pfizer.

\section{REFERENCES}

1 Fukuoka M, Yano S, Giaccone G, et al. Multi-institutional randomized phase II trial of gefitinib for previously treated patients with advanced non-small-cell lung cancer (The IDEAL 1 Trial) [corrected]. J Clin Oncol 2003; 21: 2237-2246.

2 Shepherd FA, Rodrigues Pereira J, Ciuleanu T, et al. Erlotinib in previously treated non-small-cell lung cancer. N Engl J Med 2005; 353: 123-132.

3 Keedy VL, Temin S, Somerfield MR, et al. American Society of Clinical Oncology provisional clinical opinion: epidermal growth factor receptor (EGFR) mutation testing for patients with advanced non-small-cell lung cancer considering first-line EGFR tyrosine kinase inhibitor therapy. J Clin Oncol 2011; 20: 2121-2127.

4 Soda M, Choi YL, Enomoto M, et al. Identification of the transforming EML4-ALK fusion gene in non-small-cell lung cancer. Nature 2007; 448: 561-566.

5 Kwak EL, Bang YJ, Camidge DR, et al. Anaplastic lymphoma kinase inhibition in non-small-cell lung cancer. N Engl J Med 2010; 363: 1693-1703.

6 Gloyn AL, Pearson ER, Antcliff JF, et al. Activating mutations in the gene encoding the ATP-sensitive potassium-channel subunit Kir6.2 and permanent neonatal diabetes. N Engl J Med 2004; 350: 1838-1849.

7 Pearson ER, Flechtner I, Njolstad PR, et al. Switching from insulin to oral sulfonylureas in patients with diabetes due to Kir6.2 mutations. N Engl J Med 2006; 355: 467-477.

8 Derichs N. Targeting a genetic defect: cystic fibrosis transmembrane conductance regulator modulators in cystic fibrosis. Eur Respir Rev 2013; 22: 58-65.

9 Ramsey BW, Davies J, McElvaney NG, et al. A CFTR potentiator in patients with cystic fibrosis and the G551D mutation. N Engl J Med 2011; 365: 1663-1672.

10 Repapi E, Sayers I, Wain LV, et al. Genome-wide association study identifies five loci associated with lung function. Nat Genet 2010; 42: $36-44$.

11 Hancock DB, Eijgelsheim M, Wilk JB, et al. Meta-analyses of genome-wide association studies identify multiple loci associated with pulmonary function. Nat Genet 2010; 42: 45-52.

12 Soler Artigas M, Loth DW, Wain LV, et al. Genome-wide association and large-scale follow up identifies 16 new loci influencing lung function. Nat Genet 2011; 43: 1082-1090.

13 Soler Artigas M, Wain LV, Repapi E, et al. Effect of five genetic variants associated with lung function on the risk of chronic obstructive lung disease, and their joint effects on lung function. Am J Respir Crit Care Med 2011; 184: 786-795.

14 Wilk JB, Shrine NR, Loehr LR, et al. Genome-wide association studies identify CHRNA5/3 and HTR4 in the development of airflow obstruction. Am J Respir Crit Care Med 2012; 186: 622-632.

15 Obeidat M, Wain LV, Shrine N, et al. A comprehensive evaluation of potential lung function associated genes in the SpiroMeta general population sample. PloS One 2011; 6: e19382.

16 Blakely JD, Hall IP. Current progress in pharmacogenetics. $\mathrm{Br} J$ Clin Pharmacol 2011; 71: 824-831. 